Please cite this article as: Markowitz, A. L. et al. (2022). Patch-clamp Recordings and Single Fiber Labeling from Spiral Ganglion Somata in Acutely Prepared Semi-intact Cochleae from Neonatal Rats. Bio-protocol 12(01): e4281. DOI: 10.21769/BioProtoc.4281.

\title{
Patch-clamp Recordings and Single Fiber Labeling from Spiral Ganglion Somata in Acutely Prepared Semi-intact Cochleae from Neonatal Rats

\author{
Alexander L. Markowitz ${ }^{1,2, \$}$, Megana R. lyer² and Radha Kalluri1, 2, 3, 4, *
}

\begin{abstract}
${ }^{1}$ Neuroscience Graduate Program, University of Southern California, Los Angeles, USA
${ }^{2}$ Caruso Department of Otolaryngology-Head and Neck Surgery, University of Southern California, Los Angeles, USA

${ }^{3}$ Zilkha Neurogenetic Institute, University of Southern California, Los Angeles, USA

${ }^{4}$ Department of Biomedical Engineering, University of Southern California, Los Angeles, USA

\$Current address: Department of Human Genetics, University of California, Los Angeles, USA

*For correspondence: radha@usc.edu
\end{abstract}

\begin{abstract}
[Abstract] Spiral ganglion neurons (SGN) are the primary neuronal pathway for transmitting sensory information from the inner ear to the brainstem. Recent studies have revealed significant biophysical and molecular diversity indicating that auditory neurons are comprised of sub-groups whose intrinsic properties contribute to their diverse functions. Previous approaches for studying the intrinsic biophysical properties of spiral ganglion neurons relied on patch-clamp and molecular analysis of cultured somata that were disconnected from their pre-synaptic hair cell partners. In the absence of the information provided by cell-to-cell connectivity, such studies could not associate biophysical diversity with functional sub-groups. Here we describe a protocol for preparing, recording, and labeling spiral ganglion neurons in a semi-intact ex-vivo preparation. In these preparations, the cell bodies of spiral ganglion neurons remain connected to their hair cell partners. The recordings are completed within 4 hours of euthanasia, alleviating concerns about whether long culture times and culture conditions change the intrinsic properties of neurons.
\end{abstract}

Keywords: Patch-clamping, Biocytin labeling, Spiral ganglion

[Background] The primary afferent neurons of the auditory system are the primary neural pathway transmitting information from the sensory periphery to the brainstem. In many neuronal systems, it is well-accepted that heterogeneity in ion channel composition shapes neuronal responses and functions. Similar hypotheses have been made for auditory afferents based on the observation that their cell bodies are heterogeneous in their complement of ion channels and intrinsic biophysical properties (e.g., Liu et al., 2014). However, long-standing technical obstacles have hampered clear tests of these hypotheses. Patch-clamp recordings from afferent terminals are limited only to sub-populations of neurons accessible by patch electrodes (Glowatzki and Fuchs, 2002; Rutherford et al., 2012). By contrast, recordings from isolated somata allow for recordings from a more representative population. However, when separated from their hair-cell partners, the somata lack the morphological markers needed for assigning them to a functional group (Davis and Liu, 2011). The techniques presented here overcome these obstacles by combining patch-clamp electrophysiology with single-cell labeling in semi- 
Please cite this article as: Markowitz, A. L. et al. (2022). Patch-clamp Recordings and Single Fiber Labeling from Spiral Ganglion Somata in Acutely Prepared Semi-intact Cochleae from Neonatal Rats. Bio-protocol 12(01): e4281. DOI: 10.21769/BioProtoc.4281.

intact ganglion plus epithelium preparations. The approach combines the advantages of preserving the connectivity patterns between sensory hair cells and neurons, thus allowing for associations between cell-intrinsic and functional heterogeneity. The benefits of recording from ganglion cell bodies allow for more ample sampling from a representative population of cells. Our group recently applied this method to test whether neurons with different functional properties also differ in intrinsic biophysical properties (Markowitz and Kalluri, 2020). The approach is somewhat like a cochlear slice technique described by Jagger and Housley (2003), but relies on cochlear whole mounts preparations rather than vibratome generated slices. By combining information about neuronal connectivity and intrinsic diversity, these techniques can help puzzle out how inner-ear neurons parse and encode different qualities of the sensory input.

\section{Materials and Reagents}

\section{A. Animals}

Neonatal rats [between post-natal days $(P) 3$ and $P 16$ ]

B. Dissection

1. $60 \mathrm{~mm}$ and $35 \mathrm{~mm}$ Petri dishes for transferring half heads and dissecting otic capsules

2. $10 \mathrm{~mL}$ syringes for drawing and filtering L-15/HEPES/HEPES solution

3. $0.22 \mu \mathrm{m}$ aPES membrane syringe filters (e.g., Celltreat, catalog number: 229747)

4. Insulin syringe for delivering euthanasia solution by intraperitoneal injection

5. Leibovitz medium (Sigma-Aldrich, catalog number: L4386)

6. Insulated bucket filled with ice

7. Medical grade Oxygen to dissolve into solutions (with adequate regulator and tubing). The L15/HEPES solution is not carbonate buffered and maintains a stable $\mathrm{pH}$ as $\mathrm{O}_{2}$ goes into solution.

8. Pentobarbital based euthanasia solution (e.g., Fatal Plus. $50-60 \mathrm{mg} / \mathrm{kg}$ dosing)

9. Trypsin (Sigma-Aldrich, catalog number: T1426)

10. Collagenase (Sigma-Aldrich, catalog number: C5138)

11. L-15/HEPES solution is Leibovitz medium supplemented with $10 \mathrm{mM}$ HEPES (see Recipes). The HEPES buffer stabilizes the $\mathrm{pH}$ of the L-15 medium during dissection. In all instances in this protocol, L-15/HEPES solution refers to this HEPES buffered solution.

12. Enzyme cocktail (see Recipes)

13. Standard internal solution (see Recipes)

14. $120 \mathrm{mM}$ phosphate buffer (PB) (see Recipes)

15. $5 \mathrm{mM}$ PB (see Recipes)

16. Goat serum dilution/blocking Buffer (GSDB) (see Recipes)

17. Wash buffer (see Recipes) 
Please cite this article as: Markowitz, A. L. et al. (2022). Patch-clamp Recordings and Single Fiber Labeling from Spiral Ganglion Somata in Acutely Prepared Semi-intact Cochleae from Neonatal Rats. Bio-protocol 12(01): e4281. DOI: 10.21769/BioProtoc.4281.

C. Mounting coverslip

1. $15 \mathrm{~mm}$ round glass coverslips

2. Silicon elastomer, Sylgard 184 (Dow Corning, catalog number: 4019862)

3. Nylon stockings

Note: Any stocking where single fibers can be removed.

4. Thermal tape

Note: This is a polyester tape with heat-resistant adhesive that can be found in stores selling electronics components and soldering equipment.

D. Glass bottomed recording chamber

1. Low-profile-bath recording chamber (e.g., Warner Instruments, catalog number: 64-0236)

2. Cover glass, rectangular, \#1 thickness, $22 \times 40$ (Warner Instruments, catalog number: 64-0707)

3. Silicone grease kit for making a water-tight seal between the rectangular glass and the recording chamber (Warner Instruments, catalog number: 64-0378)

E. Recording

1. Filamented borosilicate capillaries for electrodes (e.g., Sutter Instruments, catalog number: BF140-117-10)

2. Reference cell (e.g., World Precision Instruments, catalog number: RC1T)

F. Recording solutions

1. $0.2 \mu \mathrm{m}$ aPES membrane filters (e.g., Nalgene, catalog number: 566-0020)

2. Beakers $(1,000 \mathrm{~mL}, 100 \mathrm{~mL})$

3. Volumetric flask, $100 \mathrm{~mL}$

4. Serological pipettes (10 mL, $25 \mathrm{~mL})$

5. $0.22 \mu \mathrm{m}$ PES membrane syringe filters (e.g., Celltreat, catalog number: 229747)

6. 34 gauge fused silica flexible needles (World Precision Instruments, catalog number: MF34G)

7. Parafilm (Bemis, catalog number: PM992)

8. Petri dishes, $35 \mathrm{~mm}, 60 \mathrm{~mm}$ (Fisher, catalog numbers: 08772A, 08772F)

9. Syringe (10 cc, $3 \mathrm{cc})$

10. MilliQ water, sterile Ultrapure Water, $\mathrm{R}>18.18 \mathrm{mOsm} \mathrm{cm}$ (e.g., filtered by a Millipore-Sigma water purification system)

11. Biocytin (Sigma-Aldrich, catalog number: B4261)

12. HEPES (Sigma-Aldrich, catalog number: H3375)

13. LiGTP (Sigma-Aldrich, catalog number: G5884)

14. ATPdi-sodium (Sigma-Aldrich, catalog number: A7699)

15. MgATP (Sigma-Aldrich, catalog number: A9187)

16. Na-CAMP (Sigma-Aldrich, catalog number: A6885)

17. EGTA (Sigma-Aldrich, catalog number: E0396) 
18. $\mathrm{CaCl}_{2}$ (J.T. Baker, catalog number: 1311-01)

19. $\mathrm{KCl}$ (Sigma-Aldrich, catalog number: P93333)

20. $\mathrm{MgCl}_{2}$ (Sigma-Aldrich, catalog number: M1028)

21. $\mathrm{NaCl}$ (Sigma-Aldrich, catalog number: S7653)

22. $\mathrm{NaH}_{2} \mathrm{PO}_{4}$ (Sigma-Aldrich, catalog number: $\mathrm{S} 0751$ )

23. $\mathrm{NaHCO}_{3}$ (Sigma-Aldrich, catalog number: $\mathrm{S} 6297$ )

24. Sulforhodamine 101 (Molecular Probes, catalog number: S359)

25. $1 \mathrm{~N} \mathrm{NaOH}$ to adjust the $\mathrm{pH}$ of L-15/HEPES up to $\mathrm{pH}$ of 7.35.

26. $1 \mathrm{M} \mathrm{KOH}$ to adjust the $\mathrm{pH}$ of intracellular recording solutions up to $\mathrm{pH}$ of 7.35

G. Immuno-solutions

1. PBS (Thermo Scientific, catalog number: 10010-023)

2. $\mathrm{Na}_{2} \mathrm{HPO}_{4}$ (Sigma-Aldrich, catalog number: S-100G)

3. $\mathrm{NaH}_{2} \mathrm{PO}_{4}$ (Sigma, catalog number: S-5011)

4. Normal Goat Serum (Life Technologies, catalog number: 16210-064)

5. $\mathrm{NaCl}$ (J.T. Baker, catalog number: 4058-01)

6. Triton-X100 (Sigma-Aldrich, catalog number: X100)

7. $4 \%$ Paraformaldehyde (EMS, catalog number: RT-157-4)

8. Mounting medium (Vectashield Antifade Medium, catalog number: $\mathrm{H}-1000-10$ )

9. Antibodies

a. Anti-myosin-VI-rabbit polyclonal, to label the cytoplasm of hair cells (Proteus Biosciences, catalog number: 25-6791). Used at a dilution of 1:1,000.

b. Streptavidin Alexa Fluor 488 conjugate, to label biocytin-filled neurons (Molecular Probes, catalog number: S32354). Used at a dilution of 1:200.

c. Anti-peripherin rabbit, to label type II spiral ganglion neurons. (Millipore, catalog number: AB1530). Used at a dilution of 1:500.

d. Secondary antibodies include Alexa Fluor 594 anti-rabbit (Thermo Fisher Scientific, catalog number: A-11080). Used at a dilution of 1:200.

\section{Equipment}

1. Pipettors $(1,000 \mu \mathrm{L}, 200 \mu \mathrm{L}, 10 \mu \mathrm{L})$

2. Stereo Dissection Microscope (e.g., Zeiss, model: Stemi 2000)

3. Epi-illumination light source (e.g., Zeiss CL 1500 ECO)

4. Dissection Tools:

Surgical scissors for bisecting heads (e.g., straight scissors; FST, catalog number: 14060-09)

Spring scissors (e.g., FST Vannas Spring Scissor, $2.5 \mathrm{~mm}$ cutting edge)

Blunt forceps (e.g., FST Dumont \#3)

Fine forceps with fine shaft (e.g., FST Dumont \#55) 
Fine forceps (e.g., FST Dumont \#5)

5. Small animal guillotine for decapitation if dissecting rats older than P15

6. Antivibration table (e.g., Cleanbench Airtables from TMC)

7. An upright microscope with DIC optics for visualizing the 3-D structure of neurons in an intact preparation (e.g., Zeiss, model: AxioExaminer D.1)

8. Filter cube for examining the light emitted by Sulforhodamine 101. Sulforhodamine has an excitation wavelength of $\sim 586 \mathrm{~nm}$ and an emission wavelength of $\sim 605 \mathrm{~nm}$. Our setup has the Zeiss filter set \#20 with a bandpass excitation filter at $546 \pm 12 \mathrm{~nm}$, a transmission filter at 560 , and a bandpass emission filter between 576 and $640 \mathrm{~nm}$.

9. Fluorescence exciter lamp (e.g., XCite EXFO 120)

10. Optional: $10 \times$ or $20 \times$ water immersion objective for viewing sample to evaluate the quality of the sample quickly

This objective is useful but not necessary (e.g., Zeiss 20× "N Achroplan" 20×, 0.5 NA; 4209579900-000).

11. $63 \times$ objective suitable for dipping into salt solutions and good fluorescence transmission in the 400 to $800 \mathrm{~nm}$ range. Long working distance is useful for inserting electrodes (e.g., our recording setup has a Zeiss W Plan-Apochromat 63×/1.0 M27; 421480-9900-000)

12. Camera to display brightfield image during recording (e.g., Axiocam MRM digital camera with Axiovision software)

13. Micromanipulator capable of a wide range of travel with both coarse and fine step resolution. Our patch-clamp setup has a Sutter MP285 motorized micromanipulator with approximately 1 inch travel distance in 3 dimensions and approximately $0.2 \mu \mathrm{m}$ resolution in course movements and $40 \mathrm{~nm}$ resolution for fine movements.

14. A patch-clamping system with amplifier, digitizer, and computer-controlled software for delivering and recording signals

We used a Multiclamp 700B amplifier with a software-controlled front panel, Digidata 1440 (data acquisition digitizer), and the pClamp v10 software suite to design stimuli and record responses. The pClamp software was installed and the digitizer was controlled on a Windows 7 operating system.

15. Peristaltic pump with minimum 2 channels

This is used to continuously perfuse oxygenated solutions to the sample during recordings (e.g., Rainin 2-channel Peristaltic Pump).

16. Stereo dissection microscope (e.g., Stemi 2000, Zeiss)

17. Laboratory oven for curing the silicon-elastomer Sylgard

18. $\mathrm{pH}$ meter

19. Osmometer with standards for checking osmolality of solutions

20. Stir plate and stir bars

21. Precision Balance capable of measuring to $0.1 \mathrm{mg}$

22. Vertical electrode puller (e.g., Narishige PC-10) 
Please cite this article as: Markowitz, A. L. et al. (2022). Patch-clamp Recordings and Single Fiber Labeling from Spiral Ganglion Somata in Acutely Prepared Semi-intact Cochleae from Neonatal Rats. Bio-protocol 12(01): e4281. DOI: 10.21769/BioProtoc.4281.

23. Confocal imaging system capable of exciting and scanning Alexa Fluor 488 and Alexa Fluor 594 We used a Zeiss LSM 800 with a 60×, 1.42 numerical aperture, oil immersion objective, and a threefold zoom (focused on the IHC-SGN synapse). The scanning format was set to collect $1024 \times 1024$ pixels yielding a sampling of $0.069 \mu \mathrm{m} /$ pixel in the lateral $(X Y)$ dimension. Sections were acquired with z-steps of $0.49 \mu \mathrm{m}$ with pinholes set at 1 Airy unit. Fluorescent signals at an emission wavelength of 488 and 594 were scanned in separate channels in sequence to ensure optimal imagining of each structure.

\section{Software}

1. Camera and software for multichannel image acquisition of DIC brightfield and Rhodamine channels. Here we used Axiovision with the Axiocam MRM camera.

2. Image analysis program capable of image segmenting based on fluorescent intensity and producing 3-D fiber reconstructions from z-stack images of fluorescent signals. We used Imaris from Bitplane.

3. Software for designing, delivering, and recording patch-clamp data. We used the pClamp v10, a commercially available software package that interfaces with the Digidata 1440, a Molecular Devices data-acquisition digitizer.

\section{Procedure}

A. Prepare Ahead of Time

1. Internal solution: Prepare a large batch $(\sim 50 \mathrm{~mL})$ of the intracellular solution under sterile conditions (see Recipes). Work fast and keep the solution cold during preparation to prevent ATP, cAMP, and LiGTP degradation. Filter using 0.2- $\mu \mathrm{m}$ PES membrane syringe filter and store at $-20^{\circ} \mathrm{C}$ in $1-2.5 \mathrm{~mL}$ aliquots.

Note: Add biocytin fresh on the recording day.

2. L-15/HEPES solution: Prepare $1 \mathrm{~L}$ of Leibovitz medium supplemented with $10 \mathrm{mM}$ HEPES. Titrate $\mathrm{pH}$ to 7.35 with approximately $2 \mathrm{~mL}$ of $1 \mathrm{~N} \mathrm{NaOH}$. This solution can be prepared several days in advance, filtered, and stored in an airtight bottle for up to 1 week at $4^{\circ} \mathrm{C}$.

3. Prepare and store $120 \mathrm{mM}$ Phosphate Buffer (PB, $100 \mathrm{~mL}), 5 \mathrm{mM}$ PB $(25 \mathrm{~mL})$, wash buffer $(25 \mathrm{~mL})$, and goat serum dilution buffer (GSDB, $25 \mathrm{~mL})$. See recipes in the 'Recipes' section.

4. Mounting coverslips

a. Prepare three to five mounting coverslips (Figure 1) by stretching two nylon fibers extracted from a lady's nylon stocking onto a heavy metal plate (e.g., Newport metal plate, Figure 1A).

b. Attach the fibers to the plate using thermal tape. Slide a 13-15 mm coverslip under the stretched fibers.

Note: Coverslips should be cleaned in ethanol before making mounting coverslips.

c. Adjust the coverslip's position until nylon fibers make a "V" shape with approximately $1 \mathrm{~mm}$ 
Please cite this article as: Markowitz, A. L. et al. (2022). Patch-clamp Recordings and Single Fiber Labeling from Spiral Ganglion Somata in Acutely Prepared Semi-intact Cochleae from Neonatal Rats. Bio-protocol 12(01): e4281. DOI: 10.21769/BioProtoc.4281.

distance between the threads in the middle of the coverslip. Apply two dots of Sylgard silicone polymer to attach the fibers to the coverslip (Figure 1B).

d. Make several coverslips with slight variations in separation between the two fibers; this will give you optimum flexibility for mounting cochlear turns of different lengths.

e. Cover with a glass Petri dish to keep the coverslips free of dust while making.

f. Cure the Sylgard on the coverslips by placing the metal plate with coverslips into a laboratory oven set at approximately $45^{\circ} \mathrm{C}$. Incubate at minimum $1 \mathrm{~h}$ to cure the Sylgard. Cured Sylgard will no longer be tacky.

g. Store the mounting coverslips in a dust-free environment. For example, we store them in sterile culture dishes wrapped in parafilm.
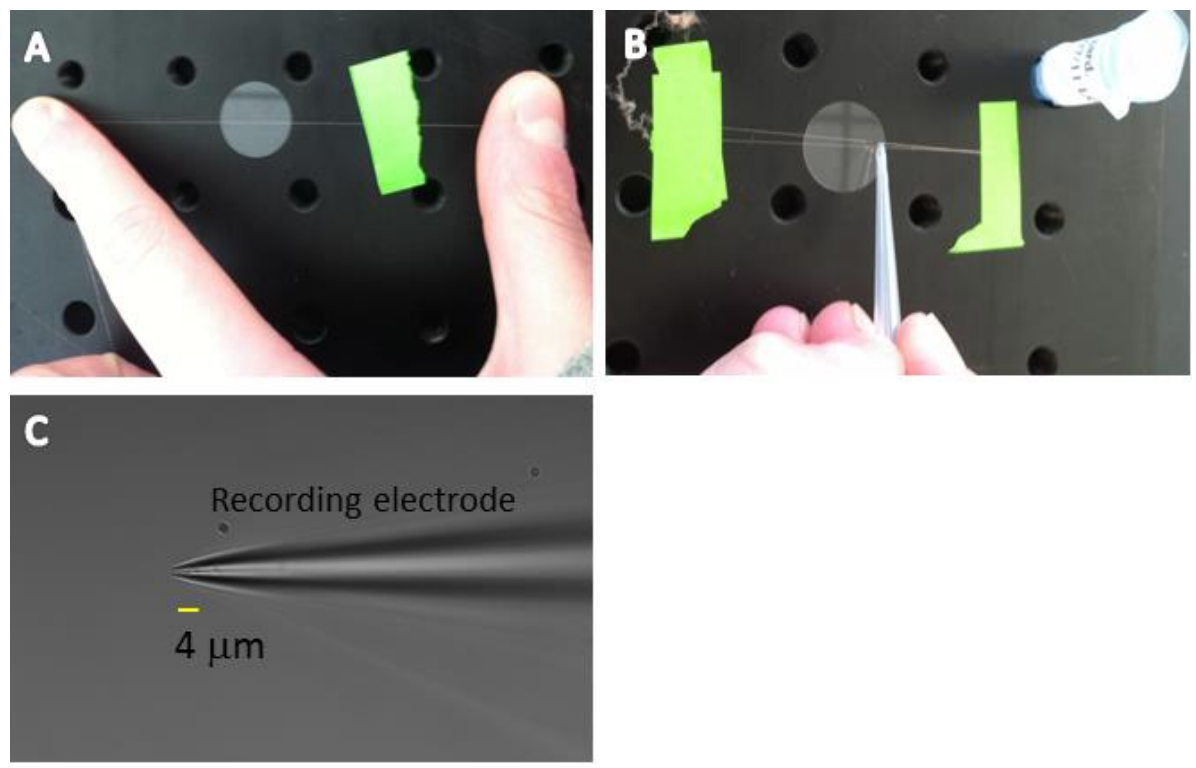

\section{Figure 1. Mounting Coverslips.}

A. Nylon fiber stretched on a $15 \mathrm{~mm}$ round glass coverslip. Use thermal tape to attach the fibers to the metal block. B. Sylgard dot applied at the edge of the coverslip to hold the two nylon fibers onto the coverslip. C. Image of a recording electrode.

\section{B. Day of recording}

1. Electrodes: Pull two or three wide-bore electrodes, five to ten cleaning electrodes, and approximately ten recording electrodes on the day of- or the night before the recording session. The settings for each electrode will vary by laboratory depending on the puller and type of glass used. In our laboratory, we make all three types of electrodes. All electrodes are made on a twostage vertical puller (Narishige PC-10) with filamented borosilicate glass. Although we outline the 'recipe' used in our laboratory in the Recipes section, puller setting should be adjusted within each laboratory and adjusted based on the type of puller. Here, the temperature settings are reported as a percentage of the maximum output provided by each stage of the two-stage pull program. The inner diameter of the recording pipette averaged approximately $1 \mu \mathrm{m}$ (Figure 1C) 
with recording pipette resistances ranging between 4.5 and $6 \mathrm{mOsm}$.

2. To reduce pipette capacitance, apply a thin hydrophobic coating to the tips of the electrodes. In our lab, we use thin sheets of parafilm wrapped around the tips or coat the tips with a silicone elastomer (Sylgard 184; Dow Corning, Midland, MI). If using Sylgard, cure it by passing the electrode through an electrically heated filament. Note, the elastomer should not be allowed to flow to the tip as this will prevent successful seals. Readers are referred to the video protocol by Brown et al. (2008) and The Sutter Pipette Cookbook as excellent resources for more tips on making suitable pipettes.

3. Dissection

a. Euthanize neonatal rats (between P3 and P16) of either sex according to institutionally approved guidelines. We anesthetize animals using an intraperitoneal injection of Fatal Plus, a pentobarbital-based euthanasia solution at a dose of $50-60 \mathrm{mg} / \mathrm{kg}$. The Fatal Plus solution is typically diluted in sterile saline to a working concentration of $50 \mathrm{mg} / \mathrm{mL}$ to allow for dosing of small rats. Decapitate once the animal reaches a deep plane of anesthesia.

b. Rinse the head in $70 \%$ ethanol followed by chilled L-15/HEPES solution.

c. Use small dissection scissors to remove the skin and cut the head along the mid-line. This will yield two half heads as in Figure 2A.

d. Remove the jaw (cut along the dashed line, Figure 2A) and scoop out the brain from each half head.

e. Transfer the two halves into $60 \mathrm{~mm}$ culture dishes filled with cold, oxygenated L-15/HEPES medium (see Recipes).

f. Remove the two otic capsules from each half-head (outlined in blue, Figure $2 \mathrm{C}$ ) using the blunt \#3 forceps. The cavity produced by the semi-circular canal is a good landmark for recognizing the top part of the otic capsule (Figure 2B-2D). Place the otic capsules into a clean dish filled with ice-cold L-15/HEPES medium. Take care during transferring because the otic capsules are partly cartilaginous and partly bony below $\mathrm{P} 8$, which makes them susceptible to crushing.

Note: The otic capsule should be kept immersed in the L-15/HEPES solution from this point forth. The L-15/HEPES solution should be replaced frequently and kept ice-cold throughout the dissection, for example by placing the dissecting dish in a large Petri dish filled with ice. 
Please cite this article as: Markowitz, A. L. et al. (2022). Patch-clamp Recordings and Single Fiber Labeling from Spiral Ganglion Somata in Acutely Prepared Semi-intact Cochleae from Neonatal Rats. Bio-protocol 12(01): e4281. DOI: 10.21769/BioProtoc.4281.

\begin{tabular}{|c|c|c|}
\hline$\because-$ & www.bio-protocol.org/e4281 & $\begin{array}{l}\text { Bio-protocol 12(01): e4281. } \\
\text { DOI:10.21769/BioProtoc. } 4281\end{array}$ \\
\hline
\end{tabular}
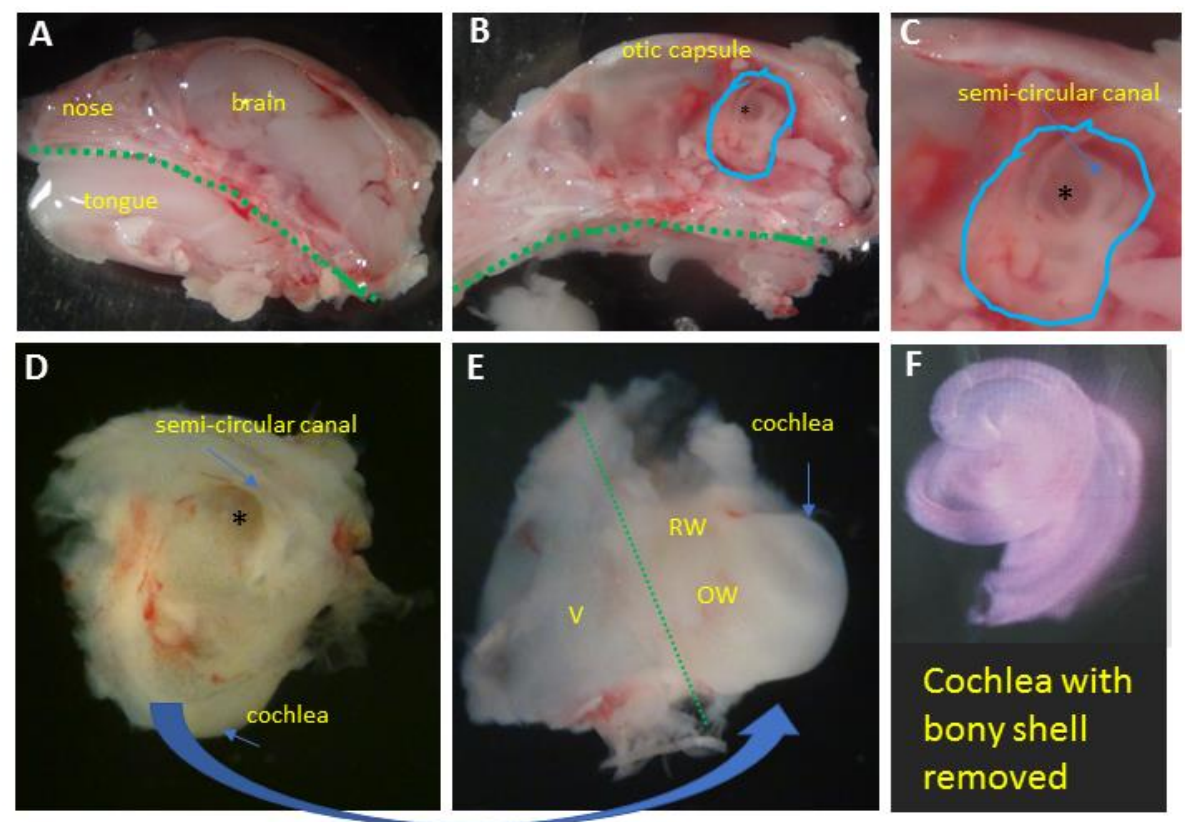

flip

Figure 2. Gross dissection to remove cochlea.

A. A bisected half head is shown with labels indicating the brain, nose, and tongue. A green line indicates the line along which a cut is made to remove the tongue. B. Half head with brain and tongue removed. Small asterisk indicates the cavity formed inside the semicircular canal. The blue line outlines the otic capsule. C. Higher magnification view of the otic capsule. D. Otic capsule removed from half head. E. Otic capsule flipped to visualize the snail-shape of the cochlea. OW - oval window, RW - round window, V - vestibular portion of the ear. F. Cochlear turns after removing the bony shell. Note the cochlea in $F$ is a different sample than from A-E.

The following steps of the cochlear dissection require considerable practice to cleanly extract the cochlear turns without damaging the neurons and the ganglion bodies. They can be mastered with consistent practice.

g. Cochleae can be identified as the spiraling snail-like portion of otic capsule (Figure 2E). Hold the cochlea in place by inserting the two arms of blunt forceps (Dumont \#3) into the round and oval windows at the base of the cochlea (alternatively hold at the vestibular portion of the inner ear).

h. Start to chip or shave off the bony casing beginning at the apex of the cochlea. Switch between fine knife scalpel and fine forceps (\#5) to remove pieces as they break off. (Note that a scalpel is not needed below P6 when the otic capsule has not fully ossified). Carefully remove all the bony casing to leave the intact cochlea with the membranous labyrinth (Figure 2E). An excellent video showing this part of the dissection can be found in Grant et al. (2010). 
Please cite this article as: Markowitz, A. L. et al. (2022). Patch-clamp Recordings and Single Fiber Labeling from Spiral Ganglion Somata in Acutely Prepared Semi-intact Cochleae from Neonatal Rats. Bio-protocol 12(01): e4281. DOI: 10.21769/BioProtoc.4281.

i. Using the fine Vannas spring scissors, cut across the spiral turns as indicated in the image (Figure 3A). This should leave three turns: apical, middle \& basal (Figure 3B).

j. Using the fine forceps (\#5), hold the middle turn tissue at the edge. Using a second pair of fine forceps (\#55), slowly pull the Stria Vascularis (Figure 3C) away from the Organ of Corti (Figure 3D). Remove the Reissner's membrane (necessary) and the tectorial membrane (optional) using fine forceps (\#55 forceps). The resulting tissue should look like Figure 3E. Note: It is important to remove Reissner's membrane as it can sometimes fall back onto the ganglion to obscure the view of the spiral ganglion somata.
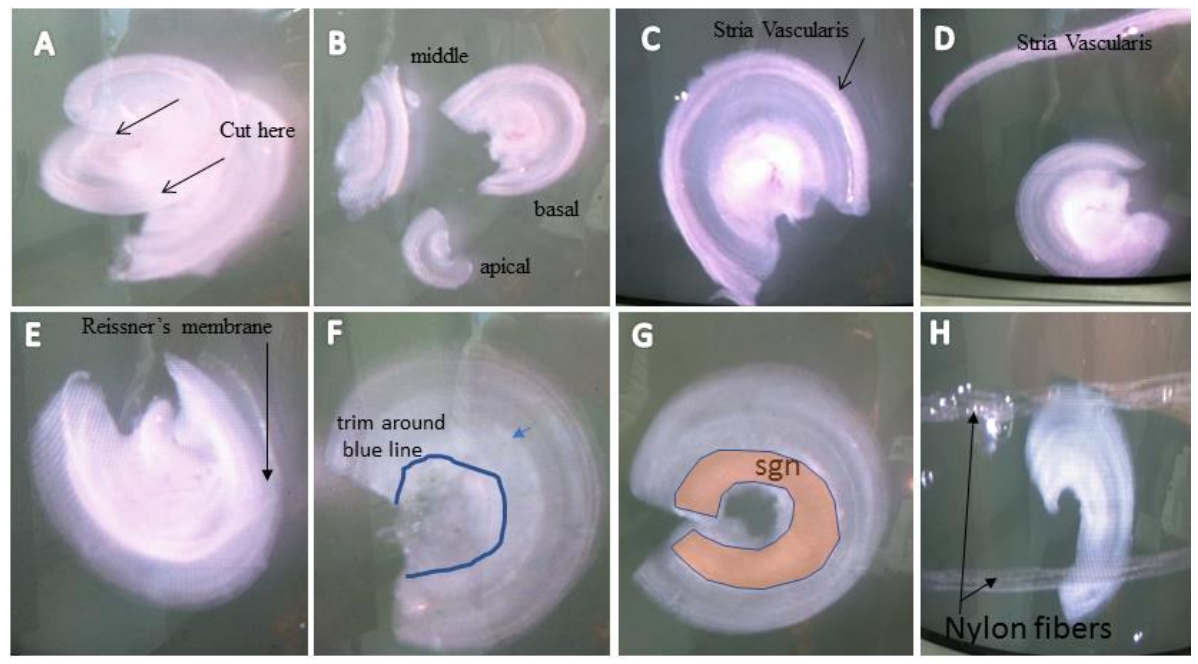

Figure 3. Fine Dissection to prepare, clean and mount the whole mount cochlear turn.

A. Cochlear spiral. B. Cochlear spiral cut into apical, middle, and basal turns. C. Middle turn with stria vascularis. D. Stria separated. E. Identifying Reissner's membrane. F. Trim line to remove fibrous core. G. Cleaned cochlear turn with spiral ganglion highlighted in orange. H. Cochlear turn mounted under nylon fibers.

k. Next, using the Vannas scissors, trim the core of the cochlear section to remove the passing fiber bundles (Figure 3F). This will allow the electrode to access the spiral ganglion neuron (SGN) cell bodies. The area to trim is easier to recognize by flipping the tissue such that the stereocilliary bundles are facing down. When so flipped, the hair cell area looks concave up. Radial fibers are also easiest to visualize when the tissue is flipped. Adjusting the epiillumination light source will help you locate the space when the ganglion region begins. The transition between the ganglion region and the inner core is brighter and will look almost like a white line (Figure 3F, blue curve drawn around that line). Cut along the blue line. The cochlea should now look like a $C$ at the end of your dissection (Figure $3 G$ ). It is now ready to be mounted and enzymatically digested. 
Please cite this article as: Markowitz, A. L. et al. (2022). Patch-clamp Recordings and Single Fiber Labeling from Spiral Ganglion Somata in Acutely Prepared Semi-intact Cochleae from Neonatal Rats. Bio-protocol 12(01): e4281. DOI: 10.21769/BioProtoc.4281.

4. Tissue mounting and enzymatic digestion

a. Prepare the recording chamber by enclosing the bottom with a vacuum-sealed rectangular cover glass. Place a mounting coverslip into the recording chamber. This will create two glass layers, the coverslip forming the bottom of the chamber and the mounting coverslip.

b. Flush the coverslip with fresh L-15/HEPES medium to remove dust.

c. To mount the cochlea into the mounting coverslip, gently lift one nylon fiber using fine forceps. It will be under considerable tension. Slide one end of the cochlear turn under the stretched fiber. Gently release the fiber onto the tissue. Take care not to allow the fiber to snap as this will cut the tissue. Lift the second fiber to slide the other end of the tissue under the second fiber. The tissue should be stretched to lie flat but do not overstretch, as this will cause the hair cell area to fold and distort. Refer to Video 1 for an example of the mounting procedure. Perform all subsequent steps, including patch-clamp recordings, immunohistochemistry, and imaging with the sample attached to the mounting coverslip (Figure $3 \mathrm{H}$ ).

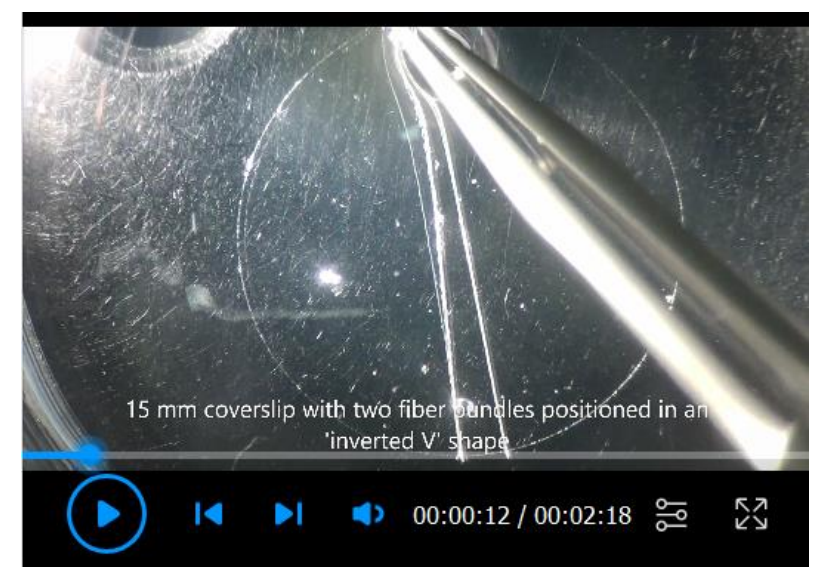

Video 1. Mounting the cochlea to coverslip for electrophysiological recording.

d. It is easiest to record from ganglion cells that are located on the edge and top of the tissue. Tissue can also be mounted with the hair bundles pointing up or down; altering the mounting direction provides access to different groups of neurons.

e. After mounting, replace the L-15/HEPES medium with the trypsin-collagenase enzyme cocktail, and place the entire recording chamber with coverslip and sample in a humidified incubator at $37^{\circ} \mathrm{C}$. Incubate for anywhere between 10 and $15 \mathrm{~min}$ (for $\sim P 6$ ). Increase the incubation time for older animals (approximately 20 min by P16). Terminate the enzymatic reaction by serially replacing the bath solution with fresh L-15/HEPES medium.

Caution: Do not attempt to adjust nylon fibers after enzymatic digestion, the tissue will tear.

Note: Although the example images show the mounted middle turn of the spiral ganglion, the preparation can come from anywhere in the cochlea, with the following caveats. In the apical turn, the ganglion forms a thick ball. The thickness of the tissue makes it harder to visualize 
Please cite this article as: Markowitz, A. L. et al. (2022). Patch-clamp Recordings and Single Fiber Labeling from Spiral Ganglion Somata in Acutely Prepared Semi-intact Cochleae from Neonatal Rats. Bio-protocol 12(01): e4281. DOI: 10.21769/BioProtoc.4281.

individual spiral ganglion somata under the microscope. In contrast, the ganglion is more spread out in the basal turn but the faster ossification of the modiolar wall makes it easier to tear neuronal fibers during the dissection (we've found it difficult to extract the basal turn cleanly after P5). The patch-electrode recordings from all regions of the cochlea become challenging as the animal ages. This is because the otic capsule continues to ossify during the first postnatal weeks, making the dissections progressively more difficult. Also, the developmental progression of myelination makes it difficult to clean the cell membrane for patch-clamping after P14.

5. Cleaning and preparing cells for patch-clamping

a. Transfer the recording chamber with the sample to the upright microscope stage and begin perfusion with oxygenated L-15/HEPES.

b. Fill a large bore electrode pulled from a glass capillary with L-15/HEPES (the electrode should have a tip diameter ranging from 5 to $10 \mu \mathrm{m}$ ). Load the electrode onto a microelectrode holder equipped with a suction line (we use the same holder as used for patchclamping). While visualizing the electrode under the $63 \times$ objective, use the manipulator to move it close to the spiral ganglion. Apply gentle suction to pull off floating debris and any cells that are lying over the ganglion cells. Avoid the temptation to pull on large sections of tissue; the enzymatic digestion makes the tissue fragile and over working the tissue will kill large numbers of cells and make the area very sticky. This cleaning step should not take the place of the cutting step where the passing fibers are removed.

c. Next, prepare individual cells for recording by peeling off the satellite (Schwann) cells that cover and myelinate the spiral ganglion somata (Figure 4A). These cells eventually create multiple layers of myelin around the cell bodies. Because the Schwann cells cling tightly to the somata, they can sometimes be hard to see. These cells need to be removed to allow recording pipettes to access the cell membrane of the neurons. To do this, bring in another series of cleaning electrodes filled with intracellular solution. First, apply positive pressure to gently puff the solution and "polish" the membranes of the Schwann cells. This polishing step often cleans the boundaries around the cell body of interest and helps one see the Schwann cell around the spiral ganglion soma. The Schwann cells usually have swelling near their nucleus. Press into the Schwann cell and apply gentle suction to pry the cell away from the neuron (Figure 4A). Take care not to stretch the neuron while pulling the Schwann cell. As the animal ages, individual SGN can have multiple Schwann cells that need to be removed. For older animals, for longer incubation durations in enzyme may be helpful (we use approximately $15 \mathrm{~min}$ at P6 and $20 \mathrm{~min}$ at P16). As long as the tip of the cleaning electrode is not blocked, the same electrode can be used to strip multiple Schwann cells. Repeat the cell stripping process until a handful of cells have been cleaned.

Note: The technique for pulling off the Schwann cells requires some practice.

d. After cleaning, allow the preparation to rest for 20 min with the perfusion system running. This rest period has the benefit of allowing the fresh L-15/HEPES to wash away loose debris. 
It also allows neurons to recover from the mechanical stress of the cleaning. Cells that are not resilient to the treatment will lyse before recording is attempted. This is often a good time to thaw and add biocytin to the intracellular solution.
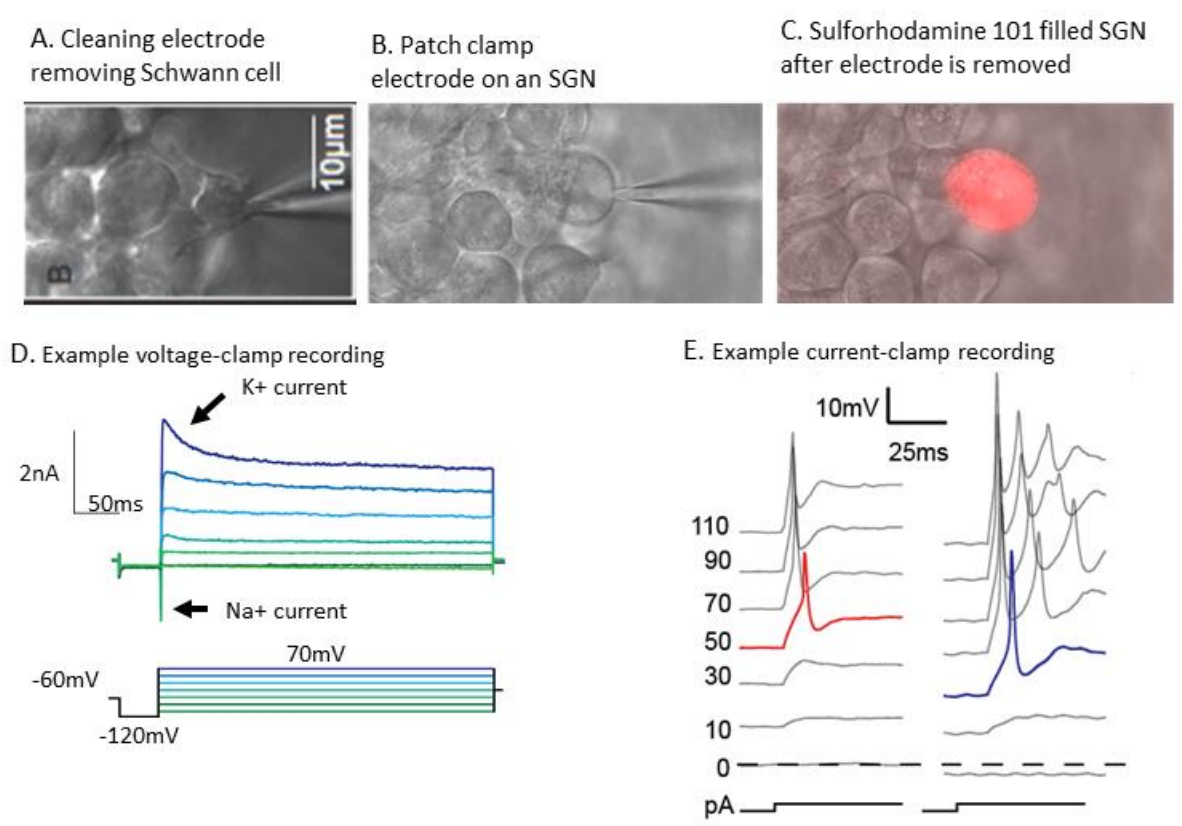

Figure 4. Patch-clamp recording and loading of single cells.

A. Cleaning electrode pulling off a Schwann cell. B. Recording electrode forming a seal on an SGN (different cell from A). C. Sulforhodamine-labeled cell as an intermediate marker to keep track of labeled cells. D. Example of whole-cell patch clamp recording in voltage clamp mode. Note the sodium and potassium currents characteristic of neurons. E. Example current clamp recordings in response to steps of current injections. The amplitude of the current step is shown next to each trace. Examples are from two different neurons showing different types of patterns of action potentials in response to the same stimulus.

6. Recording

a. Once the soma is accessible, load a recording electrode containing the intracellular solution plus biocytin (see Recipes) to record from and label the neuron. To form a successful seal on the cell, apply positive pressure to first clean and wash the area of the membrane where the electrode will land (Figure 4B). Push into the cell with slight positive pressure. Release the positive pressure. The seal will form as the positive pressure is released. Apply a little suction (negative pressure) to help with seal formation. Turn on the holding potential (we chose $-60 \mathrm{mV}$ ) as the gigaOhm seal begins to form. After a stable seal has formed, apply gentle and steady suction to rupture into the cell. The intracellular solution will flood into the cell.

b. Record voltage-gated currents in voltage clamp (Figure 4D) and firing patterns in current clamp (Figure 4E). Note the transient inward currents indicative of sodium currents and the 
Please cite this article as: Markowitz, A. L. et al. (2022). Patch-clamp Recordings and Single Fiber Labeling from Spiral Ganglion Somata in Acutely Prepared Semi-intact Cochleae from Neonatal Rats. Bio-protocol 12(01): e4281. DOI: 10.21769/BioProtoc.4281.

long-lasting outward currents indicative of potassium currents. This presence of these currents and the action potentials recorded in current clamp indicates that the recordings are from spiral ganglion neurons. More details on how to interpret these recordings can be found in Markowitz and Kalluri (2020).

c. After completing the desired recordings, we inject a series of current pulses at $10 \mathrm{~Hz}$ for $30 \mathrm{~s}$ to induce repetitive spiking from the neurons. Adjust the amplitude of the pulse train to reach current threshold. We've found that biocytin loading is enhanced in cells where we induced spiking for 30 to $40 \mathrm{~s}$. The total duration of recordings should be kept short for optimal biocytin labeling (we typically stay on a cell for less than $10 \mathrm{~min}$ ).

d. After loading the cell, turn on the epifluorescence light source to check that the cell body is brightly labeled with the Sulforhodamine (Figure 4C). A small portion of the central axon will often be labeled with Sulforhodamine, but we have not found the peripheral dendrites to label. This means that you will only know if the peripheral dendrite labels after completing the immuno-histochemistry steps.

e. Use tissue landmarks to take careful notes about the location of the recorded cell.

f. Slowly withdraw the electrode after somatic labeling is confirmed. The goal is to be sure that the cell membrane seals back up, to keep the biocytin in the cell after withdrawing the electrode. In our hands, small tip electrodes (with series resistance in the range of 5-6 mOsm) were easier to pull off than larger bore electrodes with lower resistances (1-2 mOsm). We also found that withdrawing the electrode back in a zigzag pathway was sometimes helpful for gently detaching the electrode from the cell.

g. After recordings are completed, remove the entire mounting coverslip from the recording chamber and transfer into 4\% paraformaldehyde. Allow sample to fix from 15 to $30 \mathrm{~min}$ at room temperature. All subsequent immunolabeling and imaging steps should be performed with the sample still attached to the mounting coverslip.

7. Immunohistochemistry

a. Wash three times with PBS for 15 min each. Place the samples on a shaker during the wash step. Samples can then be processed starting at the blocking step (c) or stored in PBS until ready to process. At most, we store the samples in PBS 2-3 days before starting the next step.

b. If samples were stored, wash three times in PBS for 10 min each on a shaker.

c. Blocking step: Incubate the sample for $1 \mathrm{~h}$ at room temperature $\left(\sim 25^{\circ} \mathrm{C}\right)$ in the blocking/dilution buffer consisting of $16 \%$ normal goat serum, $0.3 \%$ Triton X, $450 \mathrm{mM} \mathrm{NaCl}$, and $20 \mathrm{mM}$ phosphate buffer. The recipe for this blocking/dilution buffer is labeled as 'Goat Serum Dilution/Blocking Buffer' (GSDB).

d. Wash three times for 10 min each in wash buffer (see Recipes below).

e. Primary antibody step: Incubate samples overnight at $4^{\circ} \mathrm{C}$ with primary antibody solution. The primary antibody solution is a dilution of anti-myosin-VI-rabbit polyclonal antibody (1:1,000 dilution) and streptavidin Alexa Fluor 488 conjugate (1:200) in GSDB. The anti- 
Please cite this article as: Markowitz, A. L. et al. (2022). Patch-clamp Recordings and Single Fiber Labeling from Spiral Ganglion Somata in Acutely Prepared Semi-intact Cochleae from Neonatal Rats. Bio-protocol 12(01): e4281. DOI: 10.21769/BioProtoc.4281.

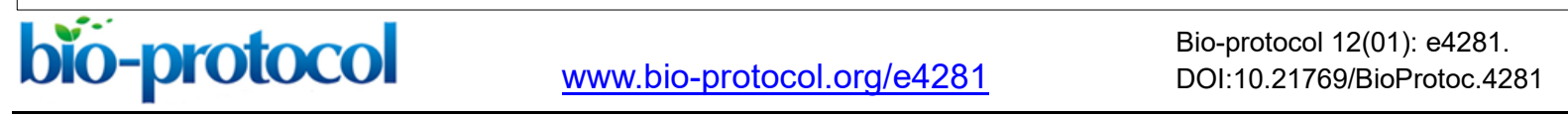

Myosin antibody labels hair cells and the streptavidin labels the biocytin-filled neurons. In some samples, we replaced the anti-Myosin antibody with the anti-peripherin (1:500) to label type II spiral ganglion neurons.

f. Wash three times for 10 min each in the wash buffer.

g. Incubate for $1 \mathrm{~h}$ at room temperature with secondary antibody Alexa Fluor 594 anti-rabbit diluted in GSDB (1:200).

h. Wash one time for $10 \mathrm{~min}$ in $5 \mathrm{mM}$ PB. The recipe for PB is found in the Recipe section below.

8. Imaging

a. Prepare slides with 4-5 hardened nail polish dots placed under the circumference of the planned coverslip. Ensure slides are dust free, use ethanol to wipe clean if needed. Place immunolabeled samples still affixed to mounting coverslip onto glass slides.

b. Add a drop of fade-protectant medium like Vectashield. Cover with another coverslip that is larger in diameter than the mounting coverslip. Alternatively, invert the mounting coverslip over a drop of Vectashield. The hardened nail polish dots prevent the coverslip from crushing the cochlear sections. Ensure that the Vectashield does not leak out of the boundaries of the coverslip.

c. Seal the edges with nail polish. Take samples to a confocal microscope for scanning [see Markowitz and Kalluri (2020) for specific examples of scan settings]. An example of a successful labeling after recording is shown in Figure 5; additional examples and strategies for analyzing these images are described (Markowitz and Kalluri, 2020). 
Please cite this article as: Markowitz, A. L. et al. (2022). Patch-clamp Recordings and Single Fiber Labeling from Spiral Ganglion Somata in Acutely Prepared Semi-intact Cochleae from Neonatal Rats. Bio-protocol 12(01): e4281. DOI: 10.21769/BioProtoc.4281.

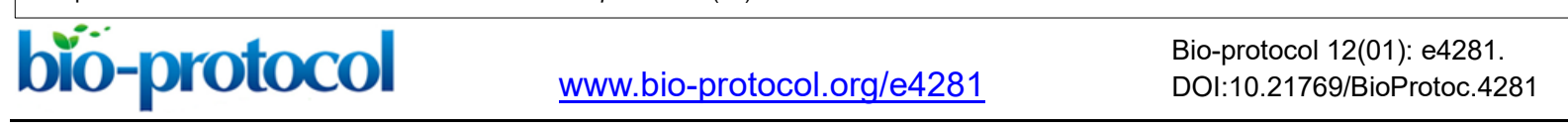

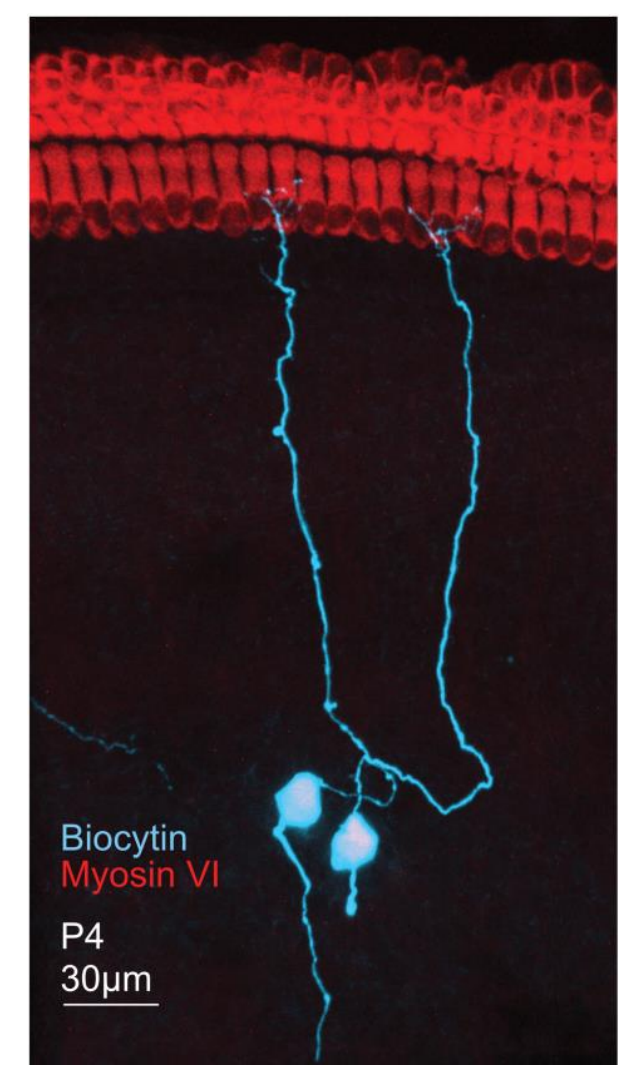

Figure 5. Two biocytin labelled spiral ganglion neurons (cyan) contacting inner hair cells (red).

\section{Data analysis}

Detailed information about data analysis can be found in Markowitz and Kalluri (2020).

\section{$\underline{\text { Recipes }}$}

\section{A. Electrodes}

All pulls are two-stage pulls with $8 \mathrm{~cm}$ and $4 \mathrm{~cm}$ slider settings. The Narishige puller has two standard weights that can be added to the puller to control the shape of the electrode.

1. Wide-bore electrode: Temperature 1: 79\%; Temperature 2: 59\%; two light weights. Break tip and firepolish to approximately $10 \mu \mathrm{m}$ tip width.

2. Cleaning electrode: Temperature 1: $79 \%$; Temperature 2: 59\%; one light weight.

3. Recording electrode: Temperature 1: $89.9 \%$; Temperature 2: $59.9 \%$, 1 light weight, Fire polish to refine shape if needed.

B. Electrophysiology Solutions

1. Standard L-15/HEPES solution

Make $1 \mathrm{~L}$ of L-15 medium supplemented with $10 \mathrm{mM}$ HEPES. 

a. Titrate with $\mathrm{NaOH}$ to adjust $\mathrm{pH}$ to 7.35 .
b. Filter through $0.2 \mu \mathrm{m}$ Nalgene filters and store in two $500-\mathrm{mL}$ sterile bottles.
C. Store unopened bottles for up to $1-2$ weeks at $4^{\circ} \mathrm{C}$.

2. Enzyme Cocktail
a. Using a $10 \mathrm{~mL}$ serological pipette, add $5 \mathrm{~mL}$ of $\mathrm{L}-15 / \mathrm{HEPES}$ solution to a clean $25 \mathrm{~mL}$ media bottle.
b. Add $0.0050 \mathrm{~g}$ of collagenase and $0.025 \mathrm{~g}$ trypsin to the bottle.
c. Add another $5 \mathrm{~mL}$ of L-15/HEPES to the bottle.
d. Add a stir bar and place on a stir plate until both enzymes are in solution.
e. The solution will be an orangish/brown.

3. Standard internal solution
$135 \mathrm{mM} \mathrm{KCl}$
$3.5 \mathrm{mM} \mathrm{MgCl}_{2}$
$3 \mathrm{mM} \mathrm{Na} 2 \mathrm{ATP}$
5 mM HEPES
5 mM EGTA
$0.1 \mathrm{mM} \mathrm{CaCl}_{2}$
$0.1 \mathrm{mM}$ Li-GTP

Add $1 \mathrm{mg} / 100 \mathrm{~mL}$ Sulforhodamine 101

Titrate with $\mathrm{KOH}$ to a $\mathrm{pH}$ of 7.35
a. Check that osmolality is approximately $280 \mathrm{mOsm}$.
b. Filter through a $0.2 \mu \mathrm{m}$ filter and store in $1-2.5 \mathrm{~mL}$ aliquots.
c. Thaw one aliquot on the day of recording.
d. Add $2.5 \mathrm{mg} / \mathrm{mL}$ biocytin to internal solution.
e. Vortex to help biocytin go completely into solution.
f. Draw solution into a $3 \mathrm{~mL}$ syringe.
g. Attach 35-gauge Microfil tip to load electrodes.
h. Keep this syringe covered in foil and on ice.

C. Immunohistochemistry Solutions

All solutions should be filtered through a $0.2 \mu \mathrm{m}$ membrane and stored at room temperature (unless otherwise specified). As with all fluorescent labeling, the solutions should be kept free of dust.

1. $120 \mathrm{mM}$ Phosphate Buffer (PB)

To make final volume of $100 \mathrm{~mL}$ :

$100 \mathrm{mM} \mathrm{Na}_{2} \mathrm{HPO}_{4} 1.412 \mathrm{~g}$

$20 \mathrm{mM} \mathrm{NaH}_{2} \mathrm{PO}_{4} 0.240 \mathrm{~g}$

MilliQ water $100 \mathrm{~mL}$

2. $5 \mathrm{mM}$ PB

To make final volume of $25 \mathrm{~mL}$ : 
Please cite this article as: Markowitz, A. L. et al. (2022). Patch-clamp Recordings and Single Fiber Labeling from Spiral Ganglion Somata in Acutely Prepared Semi-intact Cochleae from Neonatal Rats. Bio-protocol 12(01): e4281. DOI: 10.21769/BioProtoc.4281.

\begin{tabular}{|c|c|c|}
\hline 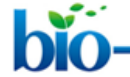 & www.bio-protocol.org/e4281 & $\begin{array}{l}\text { Bio-protocol 12(01): e4281. } \\
\text { DOI:10.21769/BioProtoc. } 4281\end{array}$ \\
\hline
\end{tabular}

PB $1.04 \mathrm{~mL}$ (see above)

MilliQ water $23.96 \mathrm{~mL}$

3. Goat Serum Dilution/Blocking Buffer (GSDB)

To make final volume of $25 \mathrm{~mL}$ :

$16 \%$ normal goat serum $4 \mathrm{~mL}$

$450 \mathrm{mM} \mathrm{NaCl} 0.6575 \mathrm{~g}$

$0.3 \%$ Triton-X100 $75 \mu \mathrm{L}$

$20 \mathrm{mM}$ PB $4.17 \mathrm{~mL}$ (see above)

MilliQ water $16.75 \mathrm{~mL}$

Note: Should result in a $\mathrm{pH}=7.4,25 \mathrm{~mL}$ final volume; store in $0.5 \mathrm{~mL}$ aliquots at $-20^{\circ} \mathrm{C}$.

4. Wash Buffer

To make final volume of $25 \mathrm{~mL}$ :

$450 \mathrm{mM} \mathrm{NaCl} 0.6575 \mathrm{~g}$

$20 \mathrm{mM}$ PB $4.17 \mathrm{~mL}$

$0.3 \%$ Triton-X $75 \mu \mathrm{L}$

MilliQ water $20.75 \mathrm{~mL}$

\section{Acknowledgments}

These techniques were developed due to the support provided by research funds from the former House Research Institute, University of Southern California, and the NIH NIDCD grants R03 DC012652 and R01 DC015512 to RK and by a graduate fellowship from the American Otological Society to AM. We acknowledge Maya Monges-Aviles and Lucy Gharibian for their early contributions to the techniques and photographs of the dissections. This protocol was derived from Markowitz and Kalluri (2020; Doi: 10.7554/eLife.55378).

\section{Competing interests}

The authors have no financial and non-financial competing interests.

\section{Ethics}

All procedures were approved by the Institutional Animal Care and Use Committee at the University of Southern California. Protocol 20704 valid until 24 February 2023.

\section{$\underline{\text { References }}$}

1. Brown, A. L., Johnson, B. E. and Goodman, M. B. (2008). Making patch-pipettes and sharp 
Please cite this article as: Markowitz, A. L. et al. (2022). Patch-clamp Recordings and Single Fiber Labeling from Spiral Ganglion Somata in Acutely Prepared Semi-intact Cochleae from Neonatal Rats. Bio-protocol 12(01): e4281. DOI: 10.21769/BioProtoc.4281.

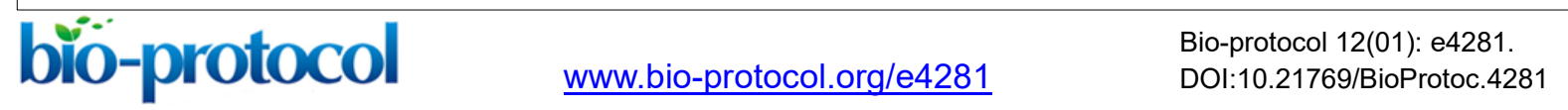

electrodes with a programmable puller. J Vis Exp (20): 939.

2. Davis, R. L. and Liu, Q. (2011). Complex primary afferents: What the distribution of electrophysiologically-relevant phenotypes within the spiral ganglion tells us about peripheral neural coding. Hear Res 276(1-2): 34-43.

3. Glowatzki, E. and Fuchs, P. (2002). Transmitter release at the hair cell ribbon synapse. Nat Neurosci 5: 147-154.

4. Liu, Q., Lee, E. and Davis, R. L. (2014). Heterogeneous intrinsic excitability of murine spiral ganglion neurons is determined by Kv1 and HCN channels. Neuroscience 257: 96-110.

5. Markowitz, A. L. and Kalluri, R. (2020). Gradients in the biophysical properties of neonatal auditory neurons align with synaptic contact position and the intensity coding map of inner hair cells. Elife 9: e55378.

6. Rutherford, M. A., Chapochnikov, N. M. and Moser, T. (2012). Spike encoding of neurotransmitter release timing by spiral ganglion neurons of the cochlea. $J$ Neurosci 32(14): 4773-4789. 